\title{
Choice of induction regimens on the risk of cytomegalovirus infection in donor-positive and recipient-negative kidney transplant recipients
}

\author{
F.L. Luan, M. Samaniego, M. Kommareddi, J.M. Park, A.O. Ojo. Choice \\ of induction regimens on the risk of cytomegalovirus infection in \\ donor-positive and recipient-negative kidney transplant recipients. \\ Transpl Infect Dis 2010: 12: 473-479. All rights reserved
}

Abstract: Background. Late occurrence of cytomegalovirus (CMV) infection remains a concern in CMV-seronegative kidney and/or pancreas transplant recipients of $\mathrm{CMV}$-seropositive organs (donor positive/recipient negative, $\mathrm{D}+/ \mathrm{R}-$ ) despite the use of prophylaxis. We investigated the impact of various antibody induction regimens on $\mathrm{CMV}$ infection in this group of patients.

Methods. A total of 254 consecutive D + / R - kidney and/or pancreas transplant patients were studied. The induction agents rabbit antithymocyte globulin (rATG) or basiliximab were used according to the center practice. All patients received prophylaxis with valganciclovir (VGCV) for either 3 or 6 months. The occurrence of CMV infection was confirmed by positive DNA viremia. Multivariate Cox regression analyses were performed to determine risk factors for CMV infection. Results. The cumulative incidence of CMV infection was 58, 112, and 59 cases per 1000 patient-years for patients who received no antibody induction, induction with rATG, or basiliximab induction, respectively $(P=0.02)$. The use of rATG but not basiliximab was associated with an increased risk for CMV infection (adjusted hazard ratio [AHR] 2.13, $95 \%$ confidence interval $[\mathrm{CI}] 1.24-3.54, P=0.006)$. Acute rejection and its treatment with rATG were not associated with an increased risk for $\mathrm{CMV}$ infection when an additional course of VGCV was given following the treatment. Longer duration of prophylaxis was associated with a reduced risk for CMV infection (AHR 0.54, 95\% CI 0.33-0.87, $P=0.011$ ). Conclusions. Induction with rATG is associated with increased risk of CMV infection. Longer duration of prophylaxis is beneficial.

\author{
F.L. Luan ${ }^{1}$, M. Samaniego ${ }^{1}$, M. Kommareddi ${ }^{1}$, \\ J.M. Park' ${ }^{2}$ A.O. $0 \mathrm{jo}^{1}$ \\ ${ }^{1}$ Department of Internal Medicine, Division of Nephrology, \\ ${ }^{2}$ Department of Pharmacy Service, College of Pharmacy, \\ University of Michigan, Ann Arbor, Michigan, USA
}

Key words: cytomegalovirus; kidney transplant; pancreas transplant; donor/recipient mismatch; rabbit anti-thymocyte globulin; basiliximab; acute rejection

Correspondence to:

Fu L. Luan, 3914 Taubman Center, Box 0364, 1500 East Medical Center Drive, Ann Arbor, MI 48109-0364, USA

Tel: +17347839041

Fax: + 17349369621

E-mail: fluan@med.umich.edu

Received 4 August 2009, revised 14 October, 11 December 2009, 11 January 2010, accepted for publication 18 January 2010

DOI: 10.1111/j.1399-3062.2010.00532.x

Transpl Infect Dis 2010: 12: 473-479
Late occurrence of cytomegalovirus (CMV) infection remains one of the most common opportunistic infections in solid organ transplantation (SOT) despite availability of specific and efficacious antiviral drugs $(1,2)$. In addition to mismatched donor and recipient CMV serology (donor positive/recipient negative, $\mathrm{D}+/ \mathrm{R}-$ ), the enhanced intensity of immunosuppression, as with the use of anti-lymphocyte antibodies, is the most important risk factor $(3,4)$. CMV infection causes significant morbidity, increases mortality, and is associated with inferior transplant outcomes, particularly in kidney transplantation (5-9).

The use of rabbit anti-thymocyte globulin (rATG) or antiinterleukin (IL)-2 receptor antibodies (basiliximab and daclizumab) has increased substantially among SOT patients in the recent era. The use of these agents has resulted in reduced incidence of acute rejection. On the other hand, the use of rATG causes profound T lymphocyte depletion and has been shown to increase the risk of 
infections, particularly CMV infection $(4,10,11)$. The effect of anti-IL2 receptor antibodies on the incidence of CMV infection is less clear $(3,11-14)$. In all clinical trials comparing rATG and anti-IL-2 receptor antibodies, the risk of CMV infection, not limited to $\mathrm{D}+/ \mathrm{R}$ - patients, appears similar in some but different in other studies, often without a unified prophylaxis approach (15-17).

The current study is based on a single-center experience involving $\mathrm{D}+/ \mathrm{R}-$ kidney and/or pancreas transplant patients treated with universal valganciclovir (VGCV) prophylaxis for 3-6 months after transplantation. This retrospective study aims to compare the risk of CMV infection among patients who received no induction, induction with rATG, or induction with basiliximab.

\section{Materials and methods}

All D + / R - kidney and/or pancreas transplant recipients from March 1, 2002 to December 31, 2007 who survived through at least the period of prophylaxis with a functioning graft were included. All patients received prophylaxis with VGCV $450 \mathrm{mg}$ daily, a dose determined by the institutional protocol and adjusted according to transplant renal function, for 3 months (March 2002 to September 2005) or 6 months (October 2005 to December 2007) after transplantation, and were followed up to the time of graft loss, death, or till April 30, 2009. The study was approved by the institutional review board.

Immunosuppression regimens, including induction and maintenance, were provided according to the institutional protocols. For induction regimen, rATG (Thymoglobulin ${ }^{\circledR}$, Genzyme, Cambridge, Massachusetts, USA) or basiliximab (Simulect ${ }^{\circledR}$, Novartis, East Hanover, New Jersey, USA) were utilized where dictated by the specific center protocols. More specifically, kidney transplant patients with panel reactive antibodies titer $\geq 20$, and/or African-American racial identification were generally given rATG, whereas patients with delayed or slow graft function and panel reactive antibodies between 0 and 19 were given basiliximab. Pancreas transplant recipients were always given antibody induction: basiliximab in the early period and rATG in more recent era. For maintenance immunosuppression, a triple drug regimen, which consisted of a calcineurin inhibitor (CNI) (cyclosporine or tacrolimus), an anti-proliferative agent (mycophenolate mofetil, mammalian target of rapamycin drugs - sirolimus/everolimus, and others), and prednisone was usually used. Target trough levels for cyclosporine and tacrolimus were $150-300$ and $5-15 \mathrm{ng} / \mathrm{mL}$, respectively, during the first 3 months. Subsequently, cyclosporine and tacrolimus trough levels were maintained at $100-150$ and $5-8 \mathrm{ng} / \mathrm{mL}$, respectively. Prednisone was tapered to $10 \mathrm{mg} /$ day at about 8 weeks post transplant and remained at $5-10 \mathrm{mg}$ daily thereafter over the study period.

The primary endpoint was the incidence of CMV infection diagnosed by positive DNA viremia determination using polymerase chain reaction (PCR) technique, regardless of the presence of symptoms during the entire duration of follow-up. With the exception of one-time protocol-driven $\mathrm{CMV} / \mathrm{PCR}$ determination at the end of prophylaxis period in a few patients transplanted in the later era (October 2005 to December 2007), the greater majority of CMV/PCR testing was requested based upon clinical suspicion by transplant physicians. The test was performed on the platform of COBAS Amplicor instrument with all reagents purchased from the Roche Diagnostics (Indianapolis, Indiana, USA). The most common clinical suspicions for ordering such determination were gastroenteric symptoms, viral-like symptoms, and/or leukopenia, particularly neutropenia. In patients with symptomatic infection, the tissue diagnosis to document the presence of tissue invasion was obtained in some patients on a case-by-case basis as determined by physicians. Patients with a diagnosis of CMV infection, with or without symptoms, were treated with an additional course of either intravenous (IV) ganciclovir or oral VGCV and temporary discontinuation of anti-proliferative agents for up to $2-3$ weeks (induction therapy phase).

Episodes of acute rejection were documented through clinically indicated biopsy and classified according to Banff criteria $(18,19)$. The treatment of acute rejection followed a center-specified protocol: acute rejection of mild degree (Banff 1a or lower) was treated with a 3-day IV steroid bolus, and acute rejection of moderate-to-severe degree (Banff $1 \mathrm{~b}$ or higher) was treated with a 7-10-day course of rATG. All patients treated with rATG were given VGCV prophylaxis, $450 \mathrm{mg}$ daily and adjusted for renal function, for an additional 3 months.

Analysis of variance and $\chi^{2}$-tests were used to compare continuous and categorical variables, respectively, for baseline demographic and clinical characteristics between patients from various groups according to the type of induction regimen they received at the time of transplant. A Poisson model was utilized to compare the cumulative incidence of CMV infection among the groups. Relative risk for CMV infection was calculated using $\chi^{2}$-test as well. The Kaplan-Meier method was used to estimate the incidences of CMV infection stratified by induction agents used. Multivariate Cox proportional hazard regression analysis was used to identify the risk factors for CMV infection. Statistical significance was set at a $P$ value of $\leq 0.05$. 


\section{Results}

A total of 254 consecutive CMV D + / R - kidney and/or pancreas transplant recipients were included in this study. The median follow-up was 1236 days from the time of transplant with range between 164 and 2586 days. Among them, 96 patients received no induction, and 114 and 44 patients received induction with rATG or basiliximab, respectively. Demographic and baseline clinical characteristics of patients from the 3 groups are presented in Table 1 . Patients were comparable with respect to age, gender, hepatitis $\mathrm{C}$ virus serology, the use of expanded criteria donor kidney, incidence of acute rejection, and baseline renal function. However, the racial composition, the use of living donors, the number of pancreas transplants, the number of

\section{Demographic and baseline characteristics}

\begin{tabular}{|c|c|c|c|c|}
\hline & $\begin{array}{l}\text { Non-induction } \\
N=96\end{array}$ & $\begin{array}{l}\text { rATG } \\
N=114\end{array}$ & $\begin{array}{l}\text { Basiliximab } \\
N=44\end{array}$ & $P$ \\
\hline Age (years, mean $\pm \mathrm{SD}$ ) & $45.9 \pm 12.5$ & $43.0 \pm 12.3$ & $47.6 \pm 9.7$ & 0.06 \\
\hline Gender (male, \%) & $68(70.8)$ & $77(67.5)$ & $29(65.9)$ & 0.81 \\
\hline Race (AA, \%) & $2(2.1)$ & $35(30.7)$ & $0(0)$ & $<0.001$ \\
\hline Hepatitis C virus positivity (\%) & $3(3.1)$ & $5(4.4)$ & $1(2.3)$ & 0.62 \\
\hline Delayed graft function (\%) & $1(1.1)$ & $16(14.0)$ & 7 (15.9) & 0.002 \\
\hline Acute rejection (\%) & & & & 0.29 \\
\hline Mild $^{1}$ & $16(16.7)$ & $14(12.3)$ & $5(11.4)$ & \\
\hline Moderate/severe ${ }^{2}$ & $12(12.5)$ & $18(15.8)$ & $2(4.5)$ & \\
\hline Calcineurin inhibitors (\%) & & & & $<0.001$ \\
\hline CsA & $92(95.8)$ & $87(76.3)$ & $27(61.4)$ & \\
\hline Tac & $4(4.2)$ & $27(23.7)$ & $17(38.6)$ & \\
\hline Anti-proliferative agents (\%) & & & & 0.05 \\
\hline MMF & $85(88.5)$ & $105(92.1)$ & $41(93.2)$ & \\
\hline mTor & $4(4.2)$ & $8(7.0)$ & $3(6.8)$ & \\
\hline Others & $7(7.3)$ & $1(0.9)$ & $0(0.0)$ & \\
\hline Extended criteria donor (\%) & $12(12.5)$ & $15(13.2)$ & $6(13.6)$ & 0.98 \\
\hline Living donor (\%) & $54(56.3)$ & $52(45.6)$ & $5(11.4)$ & $<0.001$ \\
\hline Pancreas transplant (\%) & $0(0)$ & $19(16.7)$ & $8(18.2)$ & $<0.001$ \\
\hline First transplant (\%) & $89(92.7)$ & $81(71.1)$ & $38(86.4)$ & 0.001 \\
\hline Renal diagnosis (\%) & & & & 0.002 \\
\hline APKD & $12(12.5)$ & $7(6.1)$ & $6(13.6)$ & \\
\hline DM & $29(30.2)$ & $42(36.8)$ & $27(61.4)$ & \\
\hline GN & $28(29.1)$ & $27(23.7)$ & $4(9.1)$ & \\
\hline HTN & $6(6.3)$ & $16(14.1)$ & $0(0.0)$ & \\
\hline Others & $21(21.9)$ & $22(19.3)$ & 7 (15.9) & \\
\hline Duration of prophylaxis (\%) & & & & 0.01 \\
\hline 3 months & $61(63.5)$ & $49(43.0)$ & $25(56.8)$ & \\
\hline 6 months & $35(36.5)$ & $65(57.0)$ & $19(43.2)$ & \\
\hline Serum creatinine $(\mathrm{mg} / \mathrm{dL}$, mean $\pm \mathrm{SD})$ & $1.2 \pm 0.3$ & $1.3 \pm 0.5$ & $1.2 \pm 0.3$ & 0.12 \\
\hline Follow-up (days, mean $\pm \mathrm{SD}$ ) & $1450 \pm 600$ & $1229 \pm 590$ & $1401 \pm 654$ & 0.02 \\
\hline
\end{tabular}

Table 1 
first transplants, the cases of delayed graft function, and the use of various CNIs and anti-proliferative agents were significantly different among the groups. The overwhelming representation of African-American patients in the rATG group and pancreas transplant patients in the rATG and/or basiliximab groups reflects the institutional protocols.

During the study period, 75 cases of CMV infection $(29.5 \%)$ were documented by positive CMV viremia (Fig. 1A). Five of them were diagnosed through the one-time protocol-driven $\mathrm{CMV} / \mathrm{PCR}$ determination. The median time to CMV infection was 208 days from the time of transplant, with a range from 101 to 2025 days post transplant. Following the current recommendation guideline, 49 patients had probable or confirmed CMV gastroenteric disease $(65.3 \%)$ with or without signs of hepatitis and pancreatitis, $11 \mathrm{pa}-$ tients had CMV syndrome (14.7\%), 2 patients had CMV pneumonitis $(2.6 \%)$, and 1 patient each had nephritis $(1.3 \%)$ and retinitis $(1.3 \%)(20)$. Eleven patients $(14.7 \%)$ were without symptoms or signs suggestive of CMV disease. The tissue invasion was documented in a small fraction of patients by endoscopy, broncoscopy, renal biopsy, etc. No case of CMV infection occurred during the prophylaxis period. No case of CMV infection with negative viremia occurred in this cohort.
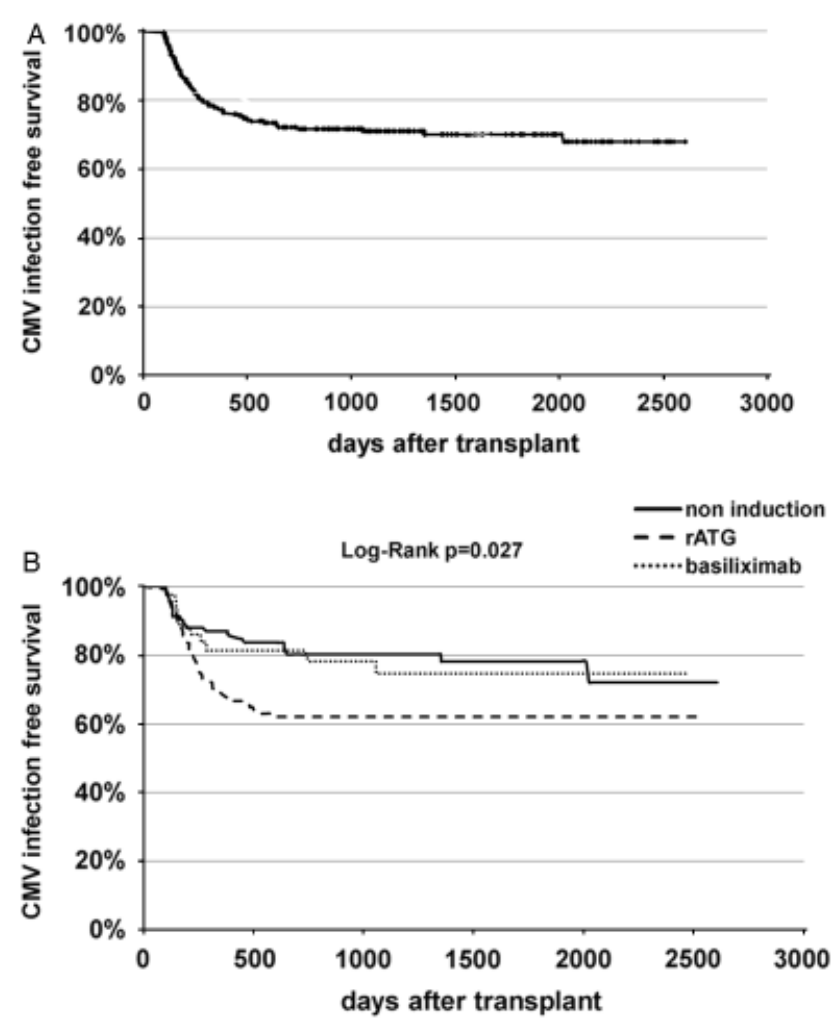

Fig. 1. (A) Overall cytomegalovirus (CMV) infection free survival, and (B) $\mathrm{CMV}$ infection free survival by induction regimens.
The cumulative incidence of CMV infection was 57, 112 , and 59 cases per 1000 patient-years follow-up among patients receiving no induction, or induction with rATG or basiliximab, respectively $(P=0.02)$. Table 2 shows the proportion of overall CMV infection between the 3 groups as well as the relative risk as determined by univariate analysis. Induction with rATG was associated with a $51 \%$ increase in the risk for CMV infection compared with no induction (risk ratio [RR] 1.51, 95\% confidence interval [CI] 1.04-2.19, $P=0.02$ ), whereas induction using basiliximab did not appear to affect the risk of CMV infection (RR 1.00, $95 \%$ CI $0.76-1.30, P=0.98)$. Kaplan-Meier survival analysis demonstrated the difference in the incidence of CMV infection among patients receiving no induction, induction with rATG, or basiliximab induction (log-rank, $P=0.027$ ) (Fig. 1B).

During the study period, 67 patients experienced 85 episodes of acute rejection. Eighteen of 75 patients with CMV infection had a total of 19 episodes of acute rejection, whereas 49 out of 179 patients without CMV infection experienced 66 episodes of acute rejection. No significant difference was seen in the incidence of acute rejection between the 2 groups $(P=0.58$ for comparison on the number of patients experiencing acute rejection, and $P=0.12$ for comparison on episodes of acute rejection, respectively). Of 18 patients with CMV infection who had experienced episodes of acute rejection, 11 patients had 11 episodes of acute rejection before the episode of CMV infection ( 5 with mild and 6 with moderate-to-severe degree of acute rejection); and 7 patients had 8 episodes of acute rejection following CMV infection ( 5 with mild and 2 with moderate-to-severe degree of acute rejection).

To determine the impact of acute rejection on the future risk of developing CMV infection, we excluded episodes of acute rejection that occurred after CMV infection among patients with a diagnosis of CMV infection. By univariate analysis, the presence of acute rejection was associated with a lower risk of CMV infection (RR $0.82,95 \%$ CI 0.70 $0.96, P=0.03)$. When we further categorized acute rejection

\begin{tabular}{|c|c|c|c|c|}
\hline $\begin{array}{l}\text { Induction } \\
\text { regimen }\end{array}$ & $\begin{array}{l}\text { CMV } \\
\text { infection (\%) }\end{array}$ & $\begin{array}{l}\text { Relative } \\
\text { risk }\end{array}$ & $95 \% \mathrm{Cl}$ & $P$ \\
\hline None $(N=96)$ & $22(22.9)$ & Ref. & $\mathrm{N} / \mathrm{A}$ & $\mathrm{N} / \mathrm{A}$ \\
\hline $\operatorname{rATG}(N=114)$ & $43(37.7)$ & 1.51 & $1.04,2.19$ & 0.02 \\
\hline Basiliximab $(N=44)$ & $10(22.7)$ & 1.00 & $0.76,1.30$ & 0.98 \\
\hline
\end{tabular}

Table 2 


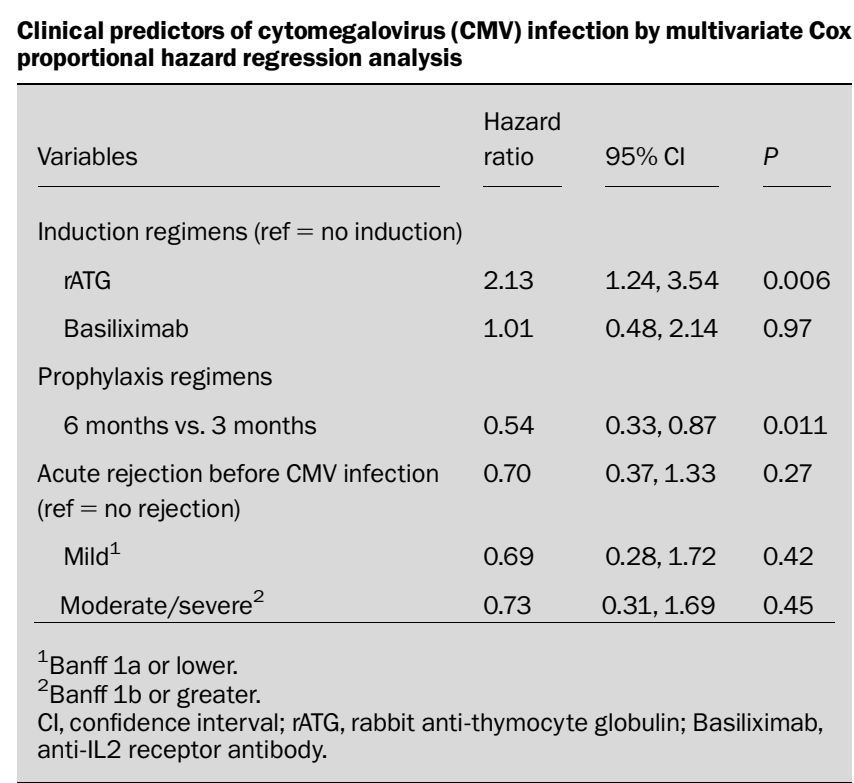

Table 3

according to its histological severity, Banff 1a or lower treated with high-dose IV steroid bolus, and Banff $1 \mathrm{~b}$ or higher treated with a course of rATG, this lower risk of developing CMV infection was no longer observed (data not shown).

Finally, we performed multivariate Cox proportional hazard regression analysis. We included all baseline covariates that showed significant difference in distribution by univariate analyses. The final model thus included the following covariates: age, race, the type of induction agents, acute rejection before $\mathrm{CMV}$ infection as a time-dependent covariate, underlying kidney disease diagnosis, the type of transplant (living vs. deceased and kidney vs. pancreas), the number of transplants, the presence of delayed graft function, the use of different CNIs and anti-proliferative agents, and the duration of prophylaxis. The use of rATG but not basiliximab was associated with a statistically significant increase in the risk for CMV infection (adjusted hazard ratio [AHR] 2.13, 95\% CI 1.24-3.54, $P=0.006$ ). Episodes of acute rejection, treated with high-dose IV steroids and/or rATG, were not associated with an increased risk of developing CMV infection during subsequent follow-up (AHR $0.70,95 \%$ CI $0.37-1.33, P=0.27$ ). Longer duration of prophylaxis with VGCVappeared associated with a significant reduction in the risk for late CMV infection (AHR 0.54, $95 \%$ CI 0.33-0.87, $P=0.011$ ) (Table 3).

\section{Discussion}

$\mathrm{D}+/ \mathrm{R}-\mathrm{CMV}$ serology mismatch and intensity of immunosuppression, particularly with the use of anti-lym- phocyte antibody for induction or treatment of acute rejection, are the 2 most important risk factors for the development of CMV infection despite the routine use of universal prophylaxis $(2-4,11,21)$. Our study here shows that the choice of induction agents impacts differently the risk of late CMV infection in $\mathrm{D}+/ \mathrm{R}$ - kidney and/or pancreas transplant recipients. In particular, the use of rATG, a T-cell depleting antibody, for induction was associated with a significant increase in the risk of CMV infection during a median follow-up of nearly 3.5 years. On the other hand, the use of basiliximab, a non-T-cell depleting antibody, did not appear to influence the risk of CMV infection. Interestingly, the use of rATG for treatment of acute rejection was not associated with increased risk of CMV infection when an additional course of VGCV was provided.

Two approaches, namely universal prophylaxis and preemptive therapy, have been variably advocated and found to be of similar efficacy in preventing symptomatic CMV infection in SOT patients across various donor and recipient CMV serology combinations. However, only universal prophylaxis has resulted in reduced CMV organ disease in $\mathrm{D}+/ \mathrm{R}$ - SOT patients (22-24). Nonetheless, the late occurrence of CMV infection remains a serious problem among this group of patients with incidence varying from $30 \%$ to as high as $48 \%(2,25)$. Our findings of $29.5 \%$ incidence rate are in line with those findings. Previous studies comparing rATG and anti-IL2 receptor antibodies have mostly focused on their clinical efficacy with CMV infection often as secondary endpoint and not limited only to $\mathrm{D}+/ \mathrm{R}$ - recipients. Furthermore, antiviral prophylaxis was not universally applied in some of these studies. Thus the results often differ from study to study $(15,16,26,27)$. Lebranchu et al. (15) showed that, in the absence of prophylaxis, the use of rATG was associated with higher risk for CMV infection than the use of basiliximab. On the other hand, Brennan et al. (16) showed opposite findings with basiliximab induced patients having more CMV infection when universal prophylaxis was provided. One of the possible explanations for their findings was the higher incidence of acute rejection in basiliximab-treated patients that required subsequent use of T-cell depleting antibodies, but no information was available regarding whether additional prophylaxis was given. Two other studies showed no difference in the risk of CMV infection between patients given rATG or basiliximab and daclizumab, although the time to the onset of CMV infection appeared shorter in patients who received rATG $(26,27)$. All these studies included patients of all donor and recipient CMV serology combinations, and only limited numbers of $\mathrm{D}+/ \mathrm{R}$ - patients.

The possible explanation of our observation rests on the fact that rATG and basiliximab have different effects on immune competent cells involved in developing anti-infectious response. While rATG is a polyclonal antibody 
causing both complement-dependent and -independent lysis of T lymphocytes (CD4 and CD8), B lymphocytes, and antigen presenting cells, basiliximab is a monoclonal antibody that binds to CD25 expressed only in activated T and B lymphocytes without causing generalized lymphocyte depletion (10,28-30). It has been well documented that specific anti-CMV immune response is a primarily $\mathrm{T}$ lymphocyte-dependent process $(31,32)$. The depletion of $\mathrm{T}$ lymphocyte population will thus result in absence of anti-CMV response and lack of CMV clearance (33).

Acute rejection is a known risk factor for CMV disease among SOT patients $(21,34,35)$. Treatment of acute rejection with enhanced immunosuppression, using either high doses of steroids or anti-lymphocytic agents, likely contributes to such enhanced risk. Most studies have not investigated the impact of different treatment modalities for acute rejection on the risk of subsequent CMV infection; and/or did not provide information about whether additional CMV prophylaxis following anti-rejection treatment was provided. In fact, one recent study by Arthurs et al. (9) showed that when prophylaxis was given after anti-rejection treatment, a lower risk of delayed-onset CMV disease was observed. Thus, the results of our study are consistent with their findings and provide additional evidence that acute rejection and its treatment do not increase the risk of subsequent CMV infection in $\mathrm{D}+/ \mathrm{R}$ - kidney and/or pancreas transplant patients when universal prophylaxis is provided at the time of transplant, and an additional course of prophylaxis is given after the use of T-cell depleting antibody for acute rejection. It is possible that increased immune response in the setting of acute rejection, in addition to the post-antirejection prophylaxis, could have contributed to the observed lower risk of CMV infection.

The major strengths of our study rely on the sample size, which to the best of our knowledge, is one of the largest involving only $\mathrm{D}+/ \mathrm{R}-$ kidney and/or pancreas transplant recipients, the number of cases with CMV infection, and long duration of follow-up with only 3 patients lost to follow-up $>345$ days after transplant. In addition, in our study the diagnosis of CMV infection was uniformly made through the determination of DNA viremia, and universal prophylaxis was provided to all study subjects. We were therefore able to directly compare no induction versus 2 different but commonly used induction agents and to assess the risk of CMV infection. Furthermore, we had detailed information on the episodes of acute rejection and its treatment and were able to estimate the impact of acute rejection and its treatment on the risk of CMV infection.

Our study suffers several limitations. First, the 3 groups of patients differed significantly in several important baseline characteristics such as racial composition, donor source, type of transplants, presence of delayed graft func- tion and maintenance immunosuppressive regimens, etc. Many of such differences in fact dictated the choice of induction agents. Although appropriate statistical approaches were utilized, certain bias and/or confounding cannot be completely excluded. Second, because the DNA viremia determination was ordered by individual physicians based on clinical suspicion, it may have under diagnosed asymptomatic cases of CMV infection, particularly if patients did not get induction and were doing well. Finally, a lower dose of VGCV was used for prophylaxis as dictated by the institutional protocol, which might have resulted in higher incidence of CMV infection in rATGtreated patients. However, a recent study did show that VGCV $450 \mathrm{mg}$ daily provided similar drug exposure compared with oral ganciclovir at $1000 \mathrm{mg} 3$ times daily in kidney transplant patients, a dose similarly effective for CMV prophylaxis $(2,36,37)$.

In conclusion, our study demonstrated elevated incidence of late occurrence of CMV infection with the use of rATG in $\mathrm{D}+/ \mathrm{R}$ - kidney and/or pancreas transplant patients following completion of universal prophylaxis. As both early and late occurrence of CMV infection is a risk factor for allograft loss and mortality, novel strategies are urgently needed to reduce such risk in this high-risk group of SOT patients receiving modern immunosuppressive regimens.

\section{References}

1. Rubin RH. Impact of cytomegalovirus infection on organ transplant recipients. Rev Infect Dis 1990; 12 (Suppl 7): S754-S766.

2. Paya C, Humar A, Dominguez E, et al. Efficacy and safety of valganciclovir vs. oral ganciclovir for prevention of cytomegalovirus disease in solid organ transplant recipients. Am J Transplant 2004; 4 (4): 611-620.

3. Mourad G, Garrigue V, Squifflet JP, et al. Induction versus noninduction in renal transplant recipients with tacrolimus-based immunosuppression. Transplantation 2001; 72 (6): 1050-1055.

4. Jamil B, Nicholls KM, Becker GJ, Walker RG. Influence of antirejection therapy on the timing of cytomegalovirus disease and other infections in renal transplant recipients. Clin Transplant 2000; 14 (1): $14-18$.

5. Abbott KC, Hypolite IO, Viola R, et al. Hospitalizations for cytomegalovirus disease after renal transplantation in the United States. Ann Epidemiol 2002; 12 (6): 402-409.

6. Sagedal S, Nordal KP, Hartmann A, et al. The impact of cytomegalovirus infection and disease on rejection episodes in renal allograft recipients. Am J Transplant 2002; 2 (9): 850-856.

7. Lewis RM, Johnson PC, Golden D, Van Buren CT, Kerman RH, Kahan $\mathrm{BD}$. The adverse impact of cytomegalovirus infection on clinical outcome in cyclosporine-prednisone treated renal allograft recipients. Transplantation 1988; 45 (2): 353-359.

8. Sagedal S, Hartmann A, Nordal KP, et al. Impact of early cytomegalovirus infection and disease on long-term recipient and kidney graft survival. Kidney Int 2004; 66 (1): 329-337. 
9. Arthurs SK, Eid AJ, Pedersen RA, et al. Delayed-onset primary cytomegalovirus disease and the risk of allograft failure and mortality after kidney transplantation. Clin Infect Dis 2008; 46 (6): 840-846.

10. Preville X, Flacher M, LeMauff B, et al. Mechanisms involved in antithymocyte globulin immunosuppressive activity in a nonhuman primate model. Transplantation 2001; 71 (3): 460-468.

11. Issa NC, Fishman JA. Infectious complications of antilymphocyte therapies in solid organ transplantation. Clin Infect Dis 2009; 48 (6): $772-786$.

12. Ozaki KS, Pestana JO, Granato CF, Pacheco-Silva A, Camargo LF. Sequential cytomegalovirus antigenemia monitoring in kidney transplant patients treated with antilymphocyte antibodies. Transpl Infect Dis 2004; 6 (2): 63-68.

13. Vincenti F, Kirkman R, Light S, et al. Interleukin-2-receptor blockade with daclizumab to prevent acute rejection in renal transplantation. Daclizumab Triple Therapy Study Group. N Engl J Med 1998; 338 (3): 161-165.

14. Lawen JG, Davies EA, Mourad G, et al. Randomized double-blind study of immunoprophylaxis with basiliximab, a chimeric antiinterleukin- 2 receptor monoclonal antibody, in combination with mycophenolate mofetil-containing triple therapy in renal transplantation. Transplantation 2003; 75 (1): 37-43.

15. Lebranchu Y, Bridoux F, Buchler M, et al. Immunoprophylaxis with basiliximab compared with antithymocyte globulin in renal transplant patients receiving MMF-containing triple therapy. Am J Transplant 2002; 2(1): 48-56.

16. Brennan DC, Daller JA, Lake KD, Cibrik D, Del Castillo D. Rabbit antithymocyte globulin versus basiliximab in renal transplantation. N Engl J Med 2006; 355 (19): 1967-1977.

17. Sollinger H, Kaplan B, Pescovitz MD, et al. Basiliximab versus antithymocyte globulin for prevention of acute renal allograft rejection. Transplantation 2001; 72 (12): 1915-1919.

18. Racusen LC, Solez K, Colvin RB, et al. The Banff 97 working classification of renal allograft pathology. Kidney Int 1999; 55 (2): 713723.

19. Racusen LC, Colvin RB, Solez K, et al. Antibody-mediated rejection criteria - an addition to the Banff 97 classification of renal allograft rejection. Am J Transplant 2003; 3 (6): 708-714.

20. Humar A, Michaels M. American Society of Transplantation recommendations for screening, monitoring and reporting of infectious complications in immunosuppression trials in recipients of organ transplantation. Am J Transplant 2006; 6 (2): 262-274.

21. San Juan R, Aguado JM, Lumbreras C, et al. Impact of current transplantation management on the development of cytomegalovirus disease after renal transplantation. Clin Infect Dis 2008; 47 (7): 875882.

22. KhouryJA, Storch GA, Bohl DL, et al. Prophylactic versus preemptive oral valganciclovir for the management of cytomegalovirus infection in adult renal transplant recipients. Am J Transplant 2006; 6 (9): 2134-2143.

23. Kalil AC, Levitsky J, Lyden E, Stoner J, Freifeld AG. Meta-analysis: the efficacy of strategies to prevent organ disease by cytomegalovirus in solid organ transplant recipients. Ann Intern Med 2005; 143 (12): 870 880.

24. Reischig T, Jindra P, Hes O, Svecova M, Klaboch J,Treska V. Valacyclovir prophylaxis versus preemptive valganciclovir therapy to prevent cytomegalovirus disease after renal transplantation. Am J Transplant 2008; 8 (1): 69-77.

25. Helantera I, Lautenschlager I, Koskinen P. Prospective follow-up of primary CMV infections after 6 months of valganciclovir prophylaxis in renal transplant recipients. Nephrol Dial Transplant 2009; 24 (1): 316-320.

26. Mattei MF, Redonnet M, Gandjbakhch I, et al. Lower risk of infectious deaths in cardiac transplant patients receiving basiliximab versus anti-thymocyte globulin as induction therapy. J Heart Lung Transplant 2007; 26 (7): 693-699.

27. Abou-Ayache R, Buchler M, Lepogamp P, et al. CMV infections after two doses of daclizumab versus thymoglobulin in renal transplant patients receiving mycophenolate mofetil, steroids and delayed cyclosporine A. Nephrol Dial Transplant 2008; 23 (6): 2024-2032.

28. Zand MS, Vo T, Huggins J, et al. Polyclonal rabbit antithymocyte globulin triggers B-cell and plasma cell apoptosis by multiple pathways. Transplantation 2005; 79 (11): 1507-1515.

29. Monti P, Allavena P, Di Carlo V, Piemonti L. Effects of antilymphocytes and anti-thymocytes globulin on human dendritic cells. Int Immunopharmacol 2003; 3 (2): 189-196.

30. Sterkers G, Baudouin V, Ansart-Pirenne H, et al. Duration of action of a chimeric interleukin-2 receptor monoclonal antibody, basiliximab, in pediatric kidney transplant recipients. Transplant Proc 2000; 32 (8): 2757-2759.

31. Walter EA, Greenberg PD, Gilbert MJ, et al. Reconstitution of cellular immunity against cy tomegalovirus in recipients of allogeneic bone marrow by transfer of T-cell clones from the donor. N Engl J Med 1995; 333 (16): 1038-1044.

32. Reusser P, Cathomas G, Attenhofer R, Tamm M,Thiel G. Cytomegalovirus (CMV)-specific Tcell immunity after renal transplantation mediates protection from CMV disease by limiting the systemic virus load. J Infect Dis 1999; 180 (2): 247-253.

33. Radha R, Jordan S, Puliyanda D, et al. Cellular immune responses to cytomegalovirus in renal transplant recipients. Am J Transplant 2005; 5 (1): 110-117.

34. Razonable RR, Rivero A, Rodriguez A, et al. Allograft rejection predicts the occurrence of late-onset cytomegalovirus (CMV) disease among CMV-mismatched solid organ transplant patients receiving prophylaxis with oral ganciclovir. J Infect Dis 2001; 184 (11): 14611464.

35. Sagedal S, Nordal KP, Hartmann A, et al. A prospective study of the natural course of cy tomegalovirus infection and disease in renal allograft recipients. Transplantation 2000; 70 (8): 1166-1174.

36. Chamberlain CE, Penzak SR, Alfaro RM, et al. Pharmacokinetics of low and maintenance dose valganciclovir in kidney transplant recipients. Am J Transplant 2008; 8 (6): 1297-1302.

37. Weng FL, Patel AM,Wanchoo R, et al. Oral ganciclovir versus low-dose valganciclovir for prevention of cytomegalovirus disease in recipients of kidney and pancreas transplants. Transplantation 2007; 83 (3): 290-296. 